\title{
ALGUNAS CARACTERÍSTICAS QUE EXPLICAN EL COMPORTAMIENTO DE LOS JÓVENES UNIVERSITARIOS HACIA EL AHORRO
}

\author{
Teresa Zamora Lobato \\ Profesora Investigadora de Negocios y Finanzas en Universidad de Xalapa \\ teresa_zamora76@hotmail.com \\ Arturo García Santillán \\ Profesor Investigador en UCC Business School Universidad Cristóbal Colón \\ agarcias@ucc.mx, arturogarciasantillan@yahoo.com.mx \\ Jésica Josefina Ramos Hernández \\ Alumna de 3er año del Doctorado en Ciencias de la Administración en \\ UCC Business School Universidad Cristóbal Colón \\ jes.jrh@gmail.com
}

Fecha de Recepción: 17 Septiembre 2018

Fecha de Admisión: 1 Octubre 2018

\section{RESUMEN}

Algunos tópicos financieros básicos como lo es el ahorro o la inversión constituyen conceptos importantes y necesarios para que la sociedad esté educada financieramente. Es por ello que el propósito del estudio se centró en identificar algunas características que explican el hábito hacia el ahorro que tienen los estudiantes universitarios, además de saber si este hábito difiere con respecto al género. Para ello fueron encuestados 60 estudiantes de la Universidad Pedagógica de Veracruz. Para el análisis de los datos se utilizaron las técnicas estadísticas de puntuaciones Z para determinar la aseveración de la proporción y la técnica ANOVA para determinar si existe diferencia de medias por género. Los principales hallazgos apuntan a que los alumnos ciertamente tienen el hábito de ahorro, aunque el importe en el que lo hacen con mayor frecuencia, es mínima la cantidad $(<$ de $\$ 3,000.00$ mensuales) y en relación a la diferencia de medias por género, únicamente se comprobó que existe diferencia en la variable INCOME, no así en las otras tres (Exp-Food, Exp-Mort-Serv y SAVE).

Palabras clave: ahorro; ingreso; estudiantes universitarios; género

\section{ABSTRACT}

SOME CHARACTERISTICS THAT EXPLAIN THE BEHAVIOR OF YOUNG UNIVERSITIES TO SAVE

Some basic financial topics such as savings and investment are important and required for a financially educated society. Therefore, the purpose of this study is to identify some characteristics 
that explain the savings habit of college students, as well as finding if this habit differs in relation to gender. For that purpose 60 students from the Universidad Pedagógica of Veracruz were surveyed and for data analysis, the statistical techniques used were $Z$ scores to test population proportion and ANOVA to determine if there are differences by gender between the means. The main findings point to students having a savings habit, even though the amounts are minimal $(<$ than $\$ 3,000.00$ MXN monthly) and regarding gender differences, it was only proven that there is a difference in the variable INCOME, but not in the other three (Exp-Food, Exp-Mort-Serv y SAVE).

Keywords: saving; entry; university students; gender

\section{1.- INTRODUCCIÓN}

El ahorro es uno de los aspectos más importantes en la vida económica de las personas, debido a que es la clave para obtener una independencia financiera y acumular riqueza. Además, el tener ahorros permite una seguridad económica y el poder cumplir metas como poner un negocio propio 0 adquirir algún bien como una vivienda 0 un automóvil. Los ahorros pueden tener diversos objetivos: ahorrar para cubrir costos de educación o servicios médicos, planeación para el retiro o poder afrontar emergencias de distinta índole.

Sin embargo, hoy en día existen opciones de ahorro limitadas, debido a que las instituciones financieras como la banca múltiple no ofrecen un producto de inversión que garantice como tal, un ahorro a largo plazo, esto debido a que la situación económica del país ha ocasionado una gran inestabilidad de los mercados financieros, como lo mencionan García-Santillán, Escalera-Chávez y Venegas-Martínez (2014).

Diversas instituciones y autores han encontrado hallazgos relevantes por medio de investigaciones sobre la falta de ahorro y la escasa visión de inversión, un ejemplo de ello es el estudio que realizó la Comisión Nacional Bancaria y de Valores (CNBV), la cual señala en su reporte unas cifras alarmantes que se refieren al dato significativo sobre la cartera vencida de créditos al consumo en México: ésta es ahora casi seis veces superior a la que existía al inicio de la década (CNBV, 2009).

Por su parte, el Banco del Ahorro Nacional y Servicios Financieros Bansefi (2009), señala que la población atendida por la banca comercial es de aproximadamente $37 \%$ de la población económicamente activa (PEA). En su mayoría, esta población se encuentra ubicada en zonas urbanas y por tanto "sólo el 13\% de los hogares rurales reportan acceso a instrumentos de depósitos de ahorro 0 crédito" (Gómez y Vega, 2006).

De esta manera, es posible afirmar que para que las disposiciones en materia de política económica funcionen, no sólo es necesario que el Estado intervenga, sino también que los ciudadanos se formen e informen, con el objeto de participar en la economía en forma responsable.

Cabe destacar que Bansefi (2009), menciona que las entidades de ahorro y crédito popular conocidas como "cajas populares" son organizaciones que por tradición se han enfocado a prestar servicios financieros a la población de menores recursos. Las cifras de Bansefi indican que el préstamo promedio de los socios de cajas de ahorro es de aproximadamente 19 mil pesos, aproximadamente 1,400 dólares americanos, los cuales son destinados a la construcción o mejora de vivienda, a los gastos del hogar, al negocio en su caso, a la educación y a los gastos de automóvil.

De acuerdo a Klaehn, Helms y Deshpande (2006), el comportamiento del ahorro se presenta en todos los niveles socio-económicos; sin embargo, gran parte de la población emplea instrumentos informales debido a una falta de acceso a instrumentos formales de ahorro en el mercado. Otro obstáculo que mencionan es el hecho de que las personas de bajos ingresos no muestran confianza en el sistema financiero y esta falta de conocimiento financiero impide al ahorro en instituciones financieras formales.

Los datos de la Encuesta Nacional de Inclusión Financiera que se llevó a cabo en 2015 mues- 
tran que la población desconoce los productos y servicios financieros que se encuentran en el mercado, dado que el $53 \%$ no sabría a dónde acudir en caso de tener un problema financiero. Específicamente con respecto al ahorro, únicamente el $44 \%$ cuenta con algún producto de ahorro formal y el $51.5 \%$ no conoce la existencia de cuentas de ahorro que no cobran comisiones.

Asimismo, los productos de ahorro más utilizados por los mexicanos son la cuenta de nómina $(63 \%)$ y la cuenta de ahorro (40\%); no obstante, el $53 \%$ de quienes tienen cuenta de nómina y el $44 \%$ de los que poseen una cuenta de ahorro desconocen las comisiones que les cobran por hacer uso de estos instrumentos. Lo anterior ocasiona que en caso de emergencia, las personas recurren a los préstamos de familiares, amigos o conocidos para obtener recursos $(70 \%)$ y la otra opción es el empeño (48\%), tratándose éstas de fuentes informales o que pueden resultar costosas.

La relación entre la educación y el comportamiento hacia el ahorro fue explorada por Yoshino, Morgan y Trinh (2017), quienes demostraron que en Japón, el nivel de educación en general y el nivel de educación financiera se relacionan de manera positiva y significativa con el ahorro y la inclusión financiera.

Por lo anterior, se puede considerar que en lo referente a los aspectos educativos, hoy en día las instituciones tienen un rol importante en la formación y capacitación, pues ante las necesidades de información que la sociedad demanda en algunos temas específicos que son necesarios en la vida cotidiana, las instituciones de educación superior (IES) instauran espacios universitarios tanto para el alumnado, como para la sociedad en lo general.

De esta manera, los sectores sociales, empresariales y gubernamentales, por citar algunos, requieren de cursos intensivos como capacitación extracurricular o de extensión y tal es el caso específico de los tópicos financieros, los cuales son considerados como detonantes para el crecimiento económico, como sucede de manera particular con el tema de Ahorro.

Posterior a este planteamiento sobre la importancia del ahorro, resulta pertinente fijar la siguiente interrogante de estudio: ¿Cuál es el hábito hacia el ahorro que tiene el estudiante universitario? ¿El hábito de ahorro en el estudiante difiere con respecto al género?, Por lo que el objetivo del estudio es identificar el hábito que tienen los estudiantes universitarios en relación al ahorro y si este difiere con respecto al género. Además podríamos pensar, que al ser estudiantes universitarios, independientemente de que no trabajan pero que si obtienen ingresos por parte de sus padres-, entonces podrían tener y desarrollar un buen hábito de ahorro. De ahí que se plantea como hipótesis de trabajo:

Ha1: El estudiante universitario tiene el hábito del ahorro

H01: El estudiante universitario no tiene el hábito del ahorro

Ha2: El hábito del ahorro en el estudiante universitario difiere en relación al género

H02: El hábito del ahorro en el estudiante universitario no difiere en relación al género

\section{REVISIÓN DE LITERATURA}

El tema del ahorro ha sido el foco de numerosas investigaciones, principalmente con respecto a la planeación para el retiro (Anderson, Baker y Robinson, 2017; Bucher-Koenen y Lusardi, 2011; Lusardi y Mitchell, 2007 y 2008; Van Rooji, Lusardi y Alessie, 2012) o el comportamiento del ahorro en relación a la educación financiera (Lusardi, 2004; Mandell, 2008; Mandell y Schmid Klein, 2009; Sabri y McDonald, 2010; Hamilton, Shobe, Murphy-Erby y Christy, 2012), además de ser considerado uno de los tópicos financieros que forman parte de las investigaciones sobre educación e inclusión financiera (Bernheim y Garret, 1996; Chen y Volpe, 1998 y 2002; Peng, Bartholomae, Fox y Cravener, 2007; Huston, 2010; Cameron, Calderwood, Cox, Lim y Yamakota, 2013; Zait y Bertea, 2015). 
Otro ejemplo de lo anterior, es el estudio de Bernheim, Garrett y Maki (2001), donde los autores señalan que la aplicación de un curso de gestión financiera en la educación básica hace que exista un efecto positivo en la toma de decisiones en la edad adulta en relación con el ahorro, por lo cual se tiende a guardar una mayor proporción de sus ingresos que en caso de no haber recibido dicha educación. Cabe señalar que utilizaron como base de datos el 56 \% de adultos no bancarizados en todo el mundo, mediante el acceso a depósitos, préstamos y leyes en el sector financiero que promovieron la inclusión financiera.

Sin embargo Mandell (2008) difiere de lo anterior, ya que de acuerdo al estudio que realizó para la Jumpstart Coalition for personal financial literacy en donde encuestó a estudiantes de último grado de preparatoria, midiendo variables como: ingreso, administración del dinero, ahorro e inversión y gasto y crédito, dicho trabajo demostró que el comportamiento financiero de los jóvenes no va a cambiar con el tiempo, de ahí que sus decisiones financieras tienen un efecto negativo en la economía, traduciéndose en una baja tasa de ahorro y de formación de capital, un bajo nivel de ahorro para el retiro y una tasa más alta de la distribución desigual del ingreso y la riqueza.

Por su parte Dupas y Robinson (2009) llevaron a cabo un experimento en Kenia, en el cual se seleccionaron al azar cuentas bancarias de trabajadores dedicados a la venta de productos (que en su mayor parte son mujeres) y los que trabajan como conductores de bici-taxis (principalmente hombres), en zonas rurales de Kenia. Dicho experimento proporciona, que a pesar de las grandes comisiones que se cobran para retirar, una parte sustancial de las mujeres (40\%) utilizaron las cuentas y fueron capaces de ahorrar más, aunque las oportunidades de ahorro que se ofrecen a las mujeres son limitadas. En este sentido los resultados involucran transcendentales barreras de ahorro e inversión para las mujeres de acuerdo a la muestra del estudio.

Por otra parte, Fazio (2009) menciona la importancia de fomentar el ahorro en la población y el uso de los servicios que ofrecen las instituciones financieras y cabe mencionar que hay evidencia que vincula el contar con una educación financiera posibilita el uso de los instrumentos de ahorro e inversión de una forma correcta.

De Bassa Scheresberg, Lusardi, y Yakoboski (2014) Ilevaron a cabo un estudio sobre las finanzas personales de los jóvenes millenials con educación universitaria e incluso, posgrado; entre sus hallazgos, se encuentra el hecho de que el $85 \%$ de los encuestados tienen una cuenta de ahorro, a pesar de lo cual se encuentran poco preparados ante emergencias económicas, lo cual parece una contradicción o bien, esto es indicativo de que el hecho de tener una cuenta de ahorro no implica que la cantidad de recursos económicos que han ahorrado sea suficiente para hacer frente a alguna emergencia.

De manera específica, el comportamiento con respecto al ahorro en jóvenes universitarios ha sido analizado en investigaciones anteriores como es el caso del estudio realizado en Malasia por Sabri y McDonald (2010), quienes encontraron que aquellos estudiantes con mayor nivel de educación financiera eran más propensos a tener mejores comportamientos sobre el ahorro y por tanto, a tener menores problemas económicos.

Una vez que se ha llevado a cabo la revisión de literatura sobre el tema del ahorro se presenta la metodología utilizada en la presente investigación.

\section{METODOLOGÍA}

Es un estudio empírico de diseño no experimental, dado que no hay manipulación de variables independientes $(X)$ que modifiquen el efecto $(Y)$, éstas son observadas tal cual sucede. De igual forma es un estudio de tipo descriptivo y de corte transversal, el cual se aborda desde el enfoque cuantitativo en la idea de determinar si tienen o no hábito para el ahorro los estudiantes universitarios, además de conocer si se presenta una diferencia con respecto al género. 


\subsection{Población y muestra}

Como resultado de la entrevista con directivos de la Universidad Popular Autónoma de Veracruz, se consideró pertinente llevar a cabo un estudio que pudiera arrojar indicios sobre los hábitos que tienen los alumnos en materia de ahorro, como un primer acercamiento para monitorear la educación financiera que prevalece en los alumnos de esa institución. Además, un dato importante que se debe resaltar, es que estos estudiantes asisten a la universidad durante el fin de semana debido a que en su mayoría trabajan y por tanto, este formato de estudio se alinea adecuadamente a sus necesidades.

El tema de Educación Financiera es un tema de actualidad en el que diversos actores de todo el mundo están sumando esfuerzos para contrarrestar la poca educación en materia financiera que las sociedades de los países están presentando; por lo tanto, en el orden mundial y en específico en el contexto nacional, se ha visualizado la necesidad de una sociedad educada financieramente, pues así será una sociedad que tome mejores decisiones financieras.

Con estos argumentos, se procedió a realizar el estudio en el cual se contó con la participación de alumnos de las carreras de: Trabajo Social, Educación Deportiva y Educación Artística. De esta forma, la determinación de la muestra es no probabilística, dado que ésta fue seleccionada por conveniencia, es decir, se toma como población los alumnos que están inscritos en las carreras previamente descritas, y que además se encontraban presentes al momento de aplicar la encuesta, que fue durante un fin de semana en el que se encontraban en clase.

Así, se lograron encuestar a 60 estudiantes, mismos que cumplieron con los dos criterios básicos de inclusión: el primero que estuvieran presentes al momento de la encuesta y el segundo que estuvieran inscritos o vigentes, es decir, sin adeudo alguno de materias de otros semestres.

\subsection{Instrumento}

El instrumento fue diseñado a partir de una serie de indicadores relacionados al Ingreso y Ahorro, así como algunos datos del perfil del encuestado. Los ítems que se consideran en este estudio se extraen del test de Zamora-Lobato (2017).

\subsection{Procedimiento de medición}

\subsubsection{Estadístico Z de aseveración de la proporción para Ha1 y Ho1}

Con respecto al hábito de Ahorro de los alumnos universitarios, se lleva a cabo la prueba de hipótesis sobre la aseveración de la proporción. En esta prueba se busca medir mediante el estadístico $Z$, las proporciones correspondientes a las respuestas sobre el hábito de ahorro. Por lo tanto, el sentido de la hipótesis de trabajo establece que en su mayoría el estudiante tiene el hábito del ahorro, caso contrario las hipótesis nulas establecen la negación a este supuesto. Por ello, para contrastar las hipótesis de trabajo, se lleva a cabo la prueba de la proporción de la aseveración (Ho: $\mathrm{p}=0.5$, Hi: $\mathrm{p}>0.5$ ). El criterio de decisión establece rechazar Ho si Z calculada $>Z$ crítica (tablas), caso contrario no rechazar.

\subsubsection{ANOVA de un factor para $\mathrm{Ha} 2$ y $\mathrm{Ho} 2$}

Para el contraste de hipótesis se lleva a cabo el procedimiento estadístico ANOVA con el fin de probar si el hábito de ahorro en los estudiantes de la UPAV difiere con respecto al género. 


\section{ANÁLISIS DE DATOS Y SU DISCUSIÓN}

Los resultados más significativos del perfil de los alumnos son: el $60 \%$ son mujeres cuya edad es por arriba de los 23 años, según lo demuestra el mayor porcentaje (55\%), y su estado civil son solteras $(68 \%)$ mismas que estudian y trabajan, al menos así lo demuestra el porcentaje más alto obtenido $(72 \%)$ y el tipo de empleo es fijo (48\%). Finalmente el núcleo familiar se integra por 1 a 3 integrantes $(48 \%)$.

\section{Variable hábito de ahorro:}

Se observa en la gráfica 1 , que el $92 \%$ de los estudiantes encuestados, ahorran muy poco, ya que la opción 1 se refiere a una cantidad menor de $\$ 3,000.00$

\section{Gráfica 1. Ahorro}

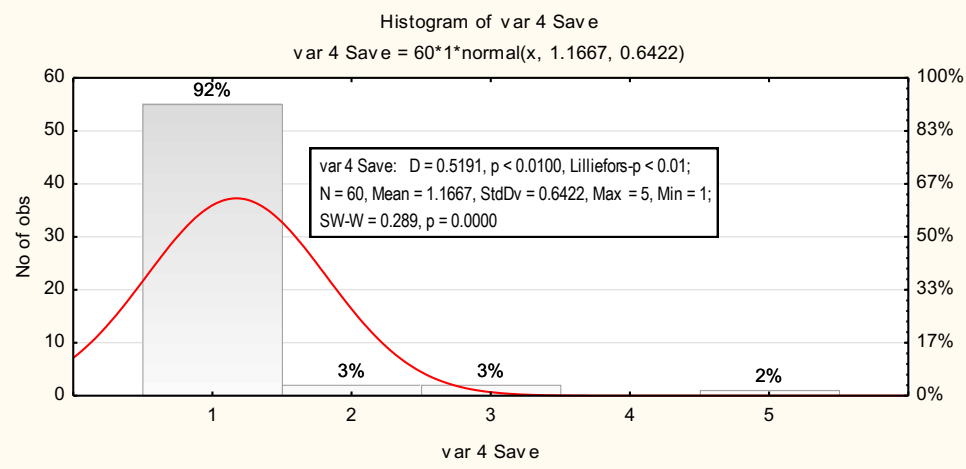

De esta forma para probar una aseveración poblacionales muy importante el estadístico de la muestra

$$
\begin{aligned}
& \begin{array}{l}
Z \\
=? \\
p=\frac{x}{n} \\
p=.5 \\
q=1-p \\
n=60
\end{array} \\
& Z_{\text {calculada }} 6.502 \text { es }>\text { a } Z_{\text {teórica }} \text { y el valor de } P=0.0001 \text { es }<\text { a la significancia } \alpha=0.05 \text {, entonces se rechaza la Ho. }
\end{aligned}
$$

Para el valor de $Z=6.502$ utilizamos de las tablas el valor de 0.999 para el área acumulativa de la izquierda del valor de Z. De ahí que el área de la derecha del estadístico de prueba Z es $1-0.9999$ $=0.0001$ y si consideramos que es $<$ al nivel de significancia $\alpha=0.05$ entonces se tiene evidencia suficiente para rechazar la hipótesis nula.

\section{ANOVA para diferencia de medias en: ingreso, gasto y hábito de ahorro por género}

En la tabla 2 se puede observar el valor del estadístico de Levene, el cual en Ingreso es menor que la significancia 0.05 , por lo que en este factor se rechazaría la igualdad de varianzas. Sin embargo, para el gasto en alimentación (Exp-Food), gasto en hipoteca (Exp-Mort-Serv) y ahorro (SAVE) la significancia es mayor a 0.05 lo que sugiere igualdad en las varianzas. 
Tabla 2. Prueba de homogeneidad de varianzas

\begin{tabular}{lrrrrr}
\hline & & & & & Sig. \\
& Estadístico de Levene & gl1 & gl2 & .010 \\
INCOME & 7.182 & 1 & 58 & .113 \\
Exp-Food & 2.583 & 1 & 58 & .147 \\
Exp-Mort-Serv & 2.157 & 1 & 58 & .395 \\
SAVE & .735 & 1 & 58 & 58 \\
\hline
\end{tabular}

En la misma idea, la tabla 3 nos muestra el ANOVA con el estadístico F y su significancia, la cual, como en la tabla 2, soporta la evidencia que en el factor INCOME se rechaza la hipótesis de igualdad de medias, no así en los factores: gasto en alimentación (Exp-Food), gasto en hipoteca (ExpMort-Serv) y ahorro (SAVE), donde la significancia es mayor a 0.05 , lo que sugiere igualdad en las varianzas.

Tabla 3. ANOVA

\begin{tabular}{llrrrrr}
\hline & & $\begin{array}{r}\text { Suma de } \\
\text { cuadrados }\end{array}$ & gl & $\begin{array}{r}\text { Media } \\
\text { cuadrática }\end{array}$ & $\mathrm{F}$ & Sig. \\
\hline INCOME & Inter-grupos & 9.344 & 1 & 9.344 & 10.104 & .002 \\
& Intra-grupos & 53.639 & 58 & .925 & & \\
& Total & 62.983 & 59 & & & \\
Exp-Food & Inter-grupos & 1.003 & 1 & 1.003 & 1.022 & .316 \\
& Intra-grupos & 56.931 & 58 & .982 & & \\
& Total & 57.933 & 59 & & & \\
Exp-Mort- & Inter-grupos & 1.600 & 1 & 1.600 & 1.619 & .208 \\
Serv & & 57.333 & 58 & .989 & & \\
& Intra-grupos & 58.933 & 59 & & & \\
SAVE & Total & .069 & 1 & .069 & .166 & .685 \\
& Inter-grupos & 24.264 & 58 & .418 & & \\
& Intra-grupos & 24.333 & 59 & & & \\
& Total & & & & &
\end{tabular}

En la tabla 3 se observa que en las dimensiones Exp-Food, Exp-Mort-Ser y SAVE el estadístico F muestra un nivel de significación mayor a 0.05, lo que nos lleva a concluir que no existen diferencias significativas por género. Sin embargo en la variable INCOME el valor de la significancia es menor a 0.05 lo que nos lleva a concluir que si hay diferencia de medias.

De esta forma, para identificar el valor teórico de $F$ con $1 \mathrm{gl}_{1}$ en el numerador y $58 \mathrm{gl}_{2}$ en el denominador, se toma el valor más cercano en tablas, siendo $60 \mathrm{gl} 2$, entonces el valor de $F$ es de 2.791 de ahí que, si tomamos el criterio de decisión entonces tenemos que:

El valor de $F$ calculada para INCOME es de $10.104>F$ crítica (2.791) por lo que se rechaza la hipótesis nula. El valor de $F$ calculada para Exp-Food es de $1.022<F$ crítica (2.791), el valor de $F$ calculada para Exp-Mort-Serv es de 1.619 < Fcrítica (2.791) en ambos casos se tiene evidencia para rechazar la hipótesis nula, en consecuencia se acepta la hipótesis alterna. Finalmente el valor de $F$ calculada para SAVE es de .116 < F crítica (2.791) por lo que no se tiene evidencia para rechazar la hipótesis nula, rechazando en consecuencia la hipótesis alterna. 


\section{DISCUSIÓN FINAL Y CONCLUSIONES}

Recordemos que el propósito del estudio se centró en medir algunos aspectos que que explican el hábito de Ahorro en el estudiante universitario; además, se estableció como propósito identificar si este comportamiento difiere en relación al género. Los hallazgos encontrados se describen a continuación.

La mayoría de los alumnos que respondieron la encuesta, fueron preponderante del género femenino, cuya edad oscila de los 23 años en adelante. Además se identificó que se encuentran solteros(as) y que están dedicados preponderantemente a su estudio y a su trabajo, este último, de tipo fijo o permanente.

El análisis ANOVA demostró que no existe una diferencia con respecto al género en las variables de gasto en alimentación, gasto en hipoteca y el más importante, el comportamiento hacia el ahorro, si bien sí existe diferencia en el ingreso que reciben, lo cual puede ser un reflejo de la disparidad laboral que existe no únicamente en México, sino en muchos otros países.

Este dato debe ser considerado con atención, ya que en diferentes estudios, se ha manifestado el rol de la mujer en varios ámbitos tanto laborales como en el estudio, ya que cada vez se están preparando más, como lo manifiesta el estudio de inclusión financiera en mujeres de la VI región (Salazar et al, 2017), en el cual se observa la participación de las mujeres en el ámbito financiero, el cual cada vez es mayor. En relación al ahorro, el estudio muestra que más del $50 \%$ de las mujeres tienen algún tipo de capacidad para el ahorro, aunque su actividad sea netamente dentro del hogar. Además, quienes poseen algún tipo de ahorro lo destinan para la vivienda, mientras un porcentaje mínimo, lo usa como un ahorro.

Si bien en esta investigación donde se aborda de manera particular el tema del ahorro no se encontró diferencia por cuestiones de género, algunos estudios sobre el nivel de educación financiera han demostrado que aún existe una disparidad entre hombres y mujeres, como el estudio de Chen y Volpe (2002), en el cual las jóvenes universitarias mostraron menor conocimiento financiero que sus contrapartes masculinas o el caso de la investigación de Moon, Ohk y Choi (2014) en China, quienes encontraron que las estudiantes universitarias tienen menores oportunidades de educación y experiencia financiera que los varones, lo que ocasiona grandes diferencias en la educación financiera con respecto al género.

Por otro lado, más de la mitad de los alumnos manifestaron obtener ingresos que no rebasan la cantidad de $\$ 3,000.00$ cada mes y finalmente, la mayoría son hijos de familia, situación que se manifiesta en alto porcentaje de respuestas (42\%), siendo este dato muy lógico, dado que el tipo de alumnos encuestados son estudiantes universitarios que aún permanecen solteros.

De esta forma podemos plasmar algunas reflexiones en torno al factor 1 que refiere el ingreso por actividad o trabajo. El análisis revela que el ingreso obtenido por el desarrollo laboral que tienen los estudiantes se avista de manera pesimista, en comparación con el informe más reciente de la Asesora Especial del Secretario General de las Naciones Unidas para el Desarrollo Financiero Incluyente, quien destaca que alrededor de 2,500 millones de adultos hoy en día se encuentran excluidos del sistema financiero. En dicho reporte, refiere el dato duro del Global Findex (2014) en el cual, en su base de datos del año 2014 señala que el $80 \%$ de quienes viven con menos de US\$2 al día, no tienen cuentas en ninguna institución financiera.

Otros ítems que se midieron fueron: gastos de alimentación y gastos de vivienda, los cuales quedaron integrados a las preguntas de la encuesta aplicada a los estudiantes objeto de estudio. En la misma idea se integraron una serie de indicadores relacionados al Ingreso y Ahorro, así como algunos datos del perfil del encuestado. Todos los ítems considerados en la encuesta, fueron extraídos del test de Zamora-Lobato (2017). 
Entre los hallazgos de la encuesta que se aplicó a los alumnos universitarios, se encuentra que éstos perciben como importante y de gran utilidad el poder contar con un presupuesto para administrar sus recursos, lográndose identificar también que el alumno percibe como importante contar con un salario más elevado para poder cubrir sus necesidades básicas.

Lo anterior coincide con la investigación de Lusardi y Mitchell (2007), en el cual los encuestados responden a varias preguntas que miden las preferencias de alfabetización y de riesgos financieros, encontrando que muchos hogares no están familiarizados con conceptos económicos necesarios para tomar decisiones financieras, de tal forma que existe un analfabetismo financiero en los jóvenes y adultos en edad avanzada, quienes no se encuentran suficientemente informados sobre conceptos financieros, lo que genera graves consecuencias para el ahorro, planes de jubilación, hipotecas y otras decisiones.

El factor IV mide el Ahorro de los Universitarios, donde se demuestra claramente que el ahorro de los universitarios es insuficiente y esto se debe a una falta de inclusión financiera, como lo mencionan otras investigaciones que tienen por objeto estudiar los niveles de conocimiento financiero, entre los que se puede destacar el de Bucher-Koenen y Lusardi (2011), quienes realizan una investigación sobre los hogares alemanes y se incluye una serie de preguntas relacionadas con conocimiento financiero y las consecuencias que tiene la falta del mismo.

También es importante considerar el impacto de los factores ambientales en el ahorro de los universitarios, como demostró el estudio de Hamilton, Shobe, Murphy-Erby y Christy (2012), quienes encontraron que si bien los padres de los jóvenes provenientes de familias de bajos recursos motivan a sus hijos a ahorrar, en estas familias se carece de ahorros para el futuro o para cumplir alguna meta en específico debido a la falta de recursos. En el caso de los estudiantes considerados en este estudio, puede observarse que sus ingresos son limitados y esto puede influir en su capacidad de ahorro.

La percepción es otro elemento fundamental que debe tenerse en cuenta con respecto al comportamiento hacia el ahorro, pues por ejemplo, si un joven tiene una percepción negativa hacia el ahorro a futuro, podríamos pensar que será menos propenso a desarrollar dicho comportamiento 0 si le brinda prioridad a sus deseos a corto plazo, será improbable que tenga ahorros de emergencia o lleve a cabo una planeación financiera a futuro (Atkinson y Messy, 2012).

El presente estudio brinda un panorama específico acerca del comportamiento hacia el ahorro en estudiantes universitarios de México y particularmente, en Veracruz, demostrando que este ahorro es aún insuficiente, además que no se presentaron diferencias significativas que se relacionen al género de la población encuestada. Otras investigaciones podrían enriquecer esta información sobre el ahorro en otros contextos, además de confirmar si verdaderamente no existen diferencias de género en otros lugares o grupos de población.

\section{REFERENCIAS}

Anderson, A., Baker, F., \& Robinson, D. T. (2017). Precautionary savings, retirement planning and misperceptions of financial literacy. Journal of Financial Economics, 126(2), 383-398.

Bansefi (Banco del Ahorro Nacional y Servicios Financieros) (2009). "Bansefi y la educación financiera". Disponible en: https://g00.gl/xuKG5r

Bernheim, B. D., \& Garrett, D. M. (1996). The determinants and consequences of financial education in the workplace: Evidence from a survey of households. National Bureau of Economic Research Working Paper No. w5667. D0I: http://dx.doi.org/10.2139/ssrn.451

Bernheim, B., M. Garrett and D. Maki, (2001). Education and saving: The long term effects of high school financial curriculum mandates. Journal of Public Economics, 80(3): 435-465.

Bucher-Koenen, T. \& Lusardi, A. (2011). Financial literacy and retirement planning in 
Germany. Journal of Pension Economics \& Finance, 10(4), 565.

Cameron, M. P., Calderwood, R., Cox, A., Lim, S., \& Yamaoka, M. (2013). Personal financial literacy among high school students in New Zealand, Japan and the USA. Citizenship, Social and Economics Education, 12(3), 200-215.

Chen, H. \& Volpe, R. P. (2002). Gender differences in personal financial literacy among college students. Financial services review, 11(3), 289.

Chen, H., \& Volpe, R. (1998). An analysis of Personal Financial Literacy Among College Students. Financial Services Review, 7(2), 107-128.

Comisión Nacional Bancaria y de Valores (CNBV). Primer Reporte de Inclusión Financiera, México, CNBV, 2009.

De Bassa Scheresberg, C., Lusardi, A., \& Yakoboski, P. J. (2014). College-educated millennials: An overview of their personal finances. TIAA-CREF Institute and the Global Financial Literacy Excellence Center,(February), 1-38.

Dupas, P., and J. Robinson (2009). Savings Constraints and Microenterprise Development: Evidence from a Field Experiment in Kenya. NBER Working Paper 14693. National Bureau of Economic Research, Cambridge, MA.

Fazio H. (2009). Crisis Mundial ¿Recesión o depresión? Chile, LOM Ediciones.

García-Santillán, A., Escalera-Chávez, M., y Venegas-Martínez, F. (2014). Matemáticas financieras, utilización de tecnología y procesos de enseñanza. ¿Cómo percibe el alumno esta trilogía? Revista Observatorio Calasanz. 6(11), 895-909.

Gómez, F. y C. González-Vega, C. (2006). "Formas de asociación cooperativa y su participación en la provisión de servicios financieros en las áreas rurales de México". Programa Finanzas Rurales. The Ohio State University. Disponible en: https://goo.gl/NpPiUe

Hamilton, L., Shobe, M., Murphy-Erby, Y. y Christy, K. (2012). "It's All About Security to Me" The Role of Environment in Youth Financial Literacy and Savings Behaviors. SAGE Open, 2(4).

Huston, S. (2010). Measuring Financial Literacy. The journal of consumer affairs, 44(2), 296-316.

INEGI y Comisión Nacional Bancaria y de Valores (2015). Encuesta Nacional de Inclusión Financiera. Recuperado de: https://goo.gl/UkhYmW

Klaehn, J., Helms, B. y Deshpande, R. (2006). México. Evaluación del ahorro a nivel nacional. Disponible en: https://g0o.gl/u1RoXp

Lusardi, A. \& Mitchell, 0. (2008). Planning and financial literacy: How do women fare?. National Bureau of Economic Research Working Paper No. w13750. DOI: http://dx.doi.org/10.3386/w13750

Lusardi, A. (2004), "Savings and the Effectiveness of Financial Education," in Mitchell, 0. and Utkus, S. (eds.), Pension Design and Structure: New Lessons from Behavioral Finance, Oxford: Oxford University Press, págs. 157-184.

Lusardi, A., \& Mitchell, 0. (2007). Financial literacy and retirement preparedness: Evidence and implications for financial education. Business economics, 42(1), 35-44.

Mandell, L. (2008). Financial education in high school. En A. Lussardi (Ed.) Overcoming the saving slump: How to increase the effectiveness of financial education and saving programs, 257-279. Chicago: The University of Chicago Press.

Mandell, L. (2008). The financial literacy of young american adults. Jumpstart coalitions survey. Recuperado de: http://www.jumpstart.org/assets/files/2008SurveyBook.pdf Sabri, M. F., \& MacDonald, M. (2010). Savings behavior and financial problems among college students: The role of financial literacy in Malaysia. Cross-Cultural Communication, 6(3), 103110. 
Mandell, L., \& Schmid Klein, L. (2009). The impact of financial literacy education on subsequent financial behavior. Journal of Financial Counseling and Planning, 20(1).

Peng, T., Bartholomae, S., Fox, J. \& Cravener, G. (2007). The impact of personal finance education delivered in high school and college courses. Journal of Family and Economic Issues, 28(2), 265-284.

Salazar, P., Denegri, M. D. C., Sepúlveda, J., Salvo, S., \& Vivallo, Ó. (2017). Estudio de inclusión financiera en mujeres de la VI Región. Instituto de Estudios Peruanos, Lima.

Van Rooij, M. C., Lusardi, A., \& Alessie, R. J. (2012). Financial literacy, retirement planning and household wealth. The Economic Journal, 122(560), 449-478.

Zait, A., \& Bertea, P. E. (2015). Financial Literacy-Conceptual Definition and Proposed Approach for a Measurement Instrument. The Journal of Accounting and Management, 4(3).

\section{ANEXOS}

\section{Instrumento}

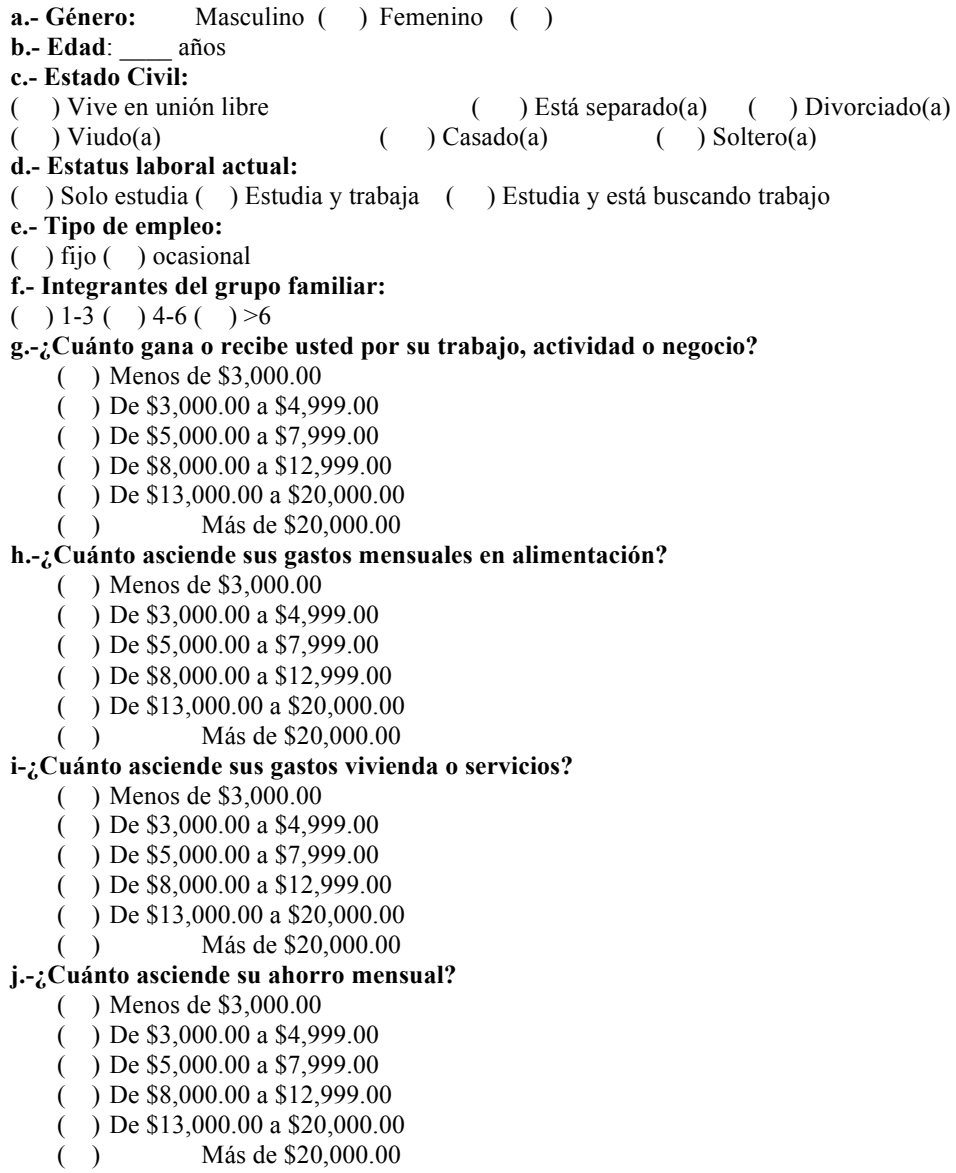


